\title{
Use of Lipofilling After Breast Cancer Conservative Surgery and Its Likely Relationship with Local Tumor Recurrence
}

\author{
Lomba-Peña $\mathrm{B}^{1}$, Peña-Fernández $\mathbf{M}^{2 *}$, Couto-González $\mathbf{I}^{3}$ and Vila-García $\mathbf{I}^{3}$ \\ ${ }^{1}$ Fellow of Family and Community Medicine, University Hospital Complex of a Coruña, Spain \\ ${ }^{2}$ Department of Gynecology and Obstetrics, Santiago de Compostela University Clinical Teaching Hospital, Spain \\ ${ }^{3}$ Department of Plastic Surgery, Santiago de Compostela University Clinical Teaching Hospital, Spain
}

*Corresponding author: Maite Peña-Fernández, Department of Gynecology and Obstetrics, Santiago de Compostela University

Clinical Teaching Hospital, Spain

\section{ARTICLE INFO}

Received: 豐 March 01, 2021

Published: 蔧 March 09, 2021

Citation: Lomba-Peña B, PeñaFernández M, Couto-González I, VilaGarcía I. Use of Lipofilling After Breast Cancer Conservative Surgery and Its Likely Relationship with Local Tumor Recurrence. Biomed J Sci \& Tech Res 34(3)-2021. BJSTR. MS.ID.005554.

Keywords: Lipofilling; Local Recurrence; Breast Cancer; Autologous Fat Grafting
ABSTRACT

Background and Purpose of Study: our study aims to determine whether the use of Lipofilling (LPF) after conservative surgery (CS) of invasive breast cancer (BC) is a possible risk factor in the local recurrence of this tumor. As secondary objectives, we will assess patient satisfaction after LPF and the percentage of patients with post-operative asymmetries.

Material and Methods: we carried out a retrospective, case-control study. We selected patients who, between 2010 and 2014, had undergone CS of Invasive BC, and were subsequently treated with LPF (using the Coleman technique) and controls had undergone CS of BC without later use of LPF. The minimum follow-up time was 5 years.

Results: No local recurrence was observed in the controls, but in the cases, 4 local recurrences were observed. This implies an incidence of $20 \%$ in CS after LPF, a statistically significant difference with a p-value of 0.0036 . The influence on recurrence of tumor size variables, vascular involvement, duration of hormone therapy and use of chemotherapy has been analyzed, without finding any statistically significant differences between cases and controls. No influence has been observed on the time elapsed from the date when CS and the first LPF and the volume of fat transferred with the risk of local recurrence. Likewise, $50 \%$ of patients are satisfied with LPF reconstruction and $20 \%$ have presented asymmetries later.

Conclusion: LPF may be a risk factor in local recurrence of invasive breast cancer after CS.

\section{Introduction}

Breast cancer (BC) is the most frequent malignant tumor among women worldwide, both in developed and developing countries. According to data published in March 2019 from Globocan 2018, the 5-year prevalence of global BC among women is estimated at $6,875,099$, ranking it as the most prevalent cancer in the world, followed by prostate cancer at 3,724,658 cases amongst men. Likewise, BC has led to 626,679 deaths worldwide in 2018, making it the leading cause of cancer death amongst women with this mortality being higher in developed countries [1]. The lifetime risk of developing BC is estimated to be approximately 1 in 8 women. Breast cancer survival has improved dramatically over the last 20 years and the survival rate from BC increases by $1.4 \%$ annually. Year after year our knowledge of the early diagnosis and treatment of $\mathrm{BC}$ improves which has allowed the overall survival rate 5 years after diagnosis of this tumor to be $82.8 \%$ in Spain (Eurocare-4), well above the European average of 79\% [2]. Since the 1980s, following the results from The National Surgical Adjuvant Breast and Bowel Project (NASBP) study, we know that in the surgical approach to 
stage I / II BC, mastectomy versus conservative surgery (CS) plus adjuvant radiotherapy do not present significant differences in terms of overall survival. However, there is a high local recurrence in the case of CS at 39\% compared with mastectomy which stands at $10 \%$ [3].

Subsequent studies were confirmed the safety of CS combined with adjuvant radiotherapy versus mastectomy in locally advanced stages after the use of adjuvant chemotherapy, with the percentage of local recurrences comparable to and less than $10 \%$ in both groups [4]. Additional research has shown that a tumor removed by CS takes longer to recur than one removed by mastectomy (3-4 years vs 2-3 years). 10 years after surgery, the risk of local recurrence is higher in breast CS compared to mastectomy (range 15\%-20\% vs $8 \%-12 \%$ ] [3]. Local tumor recurrence after CS with adjuvant radiotherapy occurs at a rate of $5 \%-15 \%$. Recurrence is most frequently observed in the original quadrant of the tumor. Recurrence at 10 years is between $10 \%$ and $20 \%$ in patients with initial stages of invasive BC. The average time to recur after the end of systemic adjuvant treatment can be 2 to 4 years or 5 to 8 years. Prognostic factors, such as tumor biology and molecular subtypes must also be considered. Thus, Luminal A or B relapse 2 or 3 times less than HER2 positive or triple negative [5].

The problem with local recurrence is that it increases the risk of distant metastasis, in addition to reducing survival by about 2 years after salvage therapy. The most important risk factors for local tumor recurrence after CS are the lack of local radiotherapy and the existence of positive lymph nodes (greater than or equal to 4 ). Other factors include the young age of the patients, the large tumor size (greater than $4 \mathrm{~cm}$ ), the high grade, the absence of hormonal receptors and lympho vascular involvement. Regarding margin involvement, it is important to point out that in situ ductal carcinomas that receive radiotherapy, a margin of $2 \mathrm{~mm}$ minimizes local recurrence and larger margins have not shown greater benefit Local recurrence is associated with better survival when it affects the mammary parenchyma but when extramammary parenchyma is affected, mortality increases [3].

In the search to improve the quality of life of women with $\mathrm{BC}$, breast reconstruction techniques are included, among which is autologous fat transfer or Lipofilling (LPF). This technique was first described in 1994 by the plastic surgeon Sydney Coleman motivated by the need to optimize the aesthetic results and manage a series of complications (volume deficit, asymmetries, superficial deformities and scar retraction) [6]. The Coleman technique consists of taking the patient's own fat tissue, purifying it and filling with it other parts of the body, such as the breast, facilitating the permanent three-dimensional modification of the anatomy. Moreover, by using the own patient's fat, the allergies and rejections are avoided. Lipofilling is a state of the art breast reconstructive technique with numerous advantages: avoids the complications of breast prostheses, improves the cutaneous stigmata of scars subjected to radiotherapy and allows a medium and long-term evolution of breasts reconstructed using autologous tissue. However, there has been some controversy with the use of $\mathrm{LPF}$ in patients with $\mathrm{BC}$, due to the potential risks of transferring autologous fat in the presence of silent tumor cells, since we know that adipose tissue secretes adipokines (leptin and growth factor of hepatocytes (HGF)), substances related to BC.

It has been shown that high serum concentrations of leptin are related to an increased risk of $\mathrm{BC}$ [7], this substance exerts cellular repair processes inducing angiogenesis processes; in the context of $\mathrm{BC}$ in in vitro studies, it has been shown that through paracrine mechanisms, fatty tissue can stimulate the growth of breast tumor cells with positive estrogen receptors (ER), also promoting mobility, migration and angiogenesis. Furthermore, leptin is overexpressed in more than $80 \%$ of malignant epithelial tumor cells and receptors for this adipokine have been detected [8]. With respect to HGF, elevated serum levels have been found in patients with BC, especially high-grade tumors, with negative ERs and in advanced clinical stages. HGF facilitates the disruption of the junctions between epithelial cells, both by autocrine and paracrine mechanisms, favoring their dispersion. In vivo it has been shown that to induce tumor angiogenesis by paracrine mechanisms and in vitro it has been demonstrated that adipocytes secreting HGF act on breast cancer cells, stimulating their proliferation [9].

Another feature to take into account is that adipose tissue contains regenerative stem cells, and by using LPF they can mimic with other tissues and generate, producing long-term benefits. However, there are also hypotheses that these stem cells can induce certain proliferative activity in the breast stroma without knowing if this would affect the development of epithelial tumors [10]. Although plausible at biological level, none these hypotheses have been demonstrated in clinical practice. The main objective of this work is therefore, to determine whether the use of LPF after a CS of BC increases the risk of local recurrence. As a secondary objective, is to determine the percentage of patient satisfaction and the percentage of asymmetries after the use of LPF.

\section{Materials and Methods}

Patients who have undergone CS for Invasive BC and subsequently LPF (cases) between January 1, 2010 and February 27, 2014, and patients who have undergone CS for BC without the use of LPF (controls) between January 1, 2010 and December 31, 2013. The inclusion criteria were age older than 18 years, follow-up of a minimum of 5 years in the Breast Unit, diagnosis of BC with CS treatment and subsequent use of radiotherapy, cases: subsequent reconstructive treatment with LPF, controls: not LPF. Exclusion criteria were no adjuvant radiotherapy treatment of the surgical bed, no follow-up in the Breast Unit within 5 years after surgical treatment, BRCA carriers, distant metastases at diagnosis, diagnosis of another type of cancer in the previous two years to the 
diagnosis and use of radiotherapy treatment in the breast prior to surgical treatment.

The LPF technique consists of a procedure under general anesthesia with a short stay of 24-72 hours. The tumescent lipoaspirate described by Klein [11] is used to take the lipograft. However, the solution described by Klein included the use of lidocaine (lidocaine $0.05 \%$, epinephrine 1: 1,000,000), to perform the technique under sedation. In our case, the wide field in which we work made the use of general anesthesia recommended. Furthermore, lidocaine has been identified as a toxic compound for adipocytes, although with a low level of evidence. Therefore, we used a lactated Ringer's solution with an adrenaline concentration of 1 : $1,000,000$ (1mg of adrenaline in $1 \mathrm{~L}$ ). This solution was infiltrated into the subcutaneous cellular tissue of the graft donor anatomical region, using multi- perforated $2 \mathrm{~mm}$ diameter Coleman infiltration cannulas. The incisions used for the infiltration of Klein's solution were the same as for the liposuction, the most common being two on both anterior superior iliac spines and at 12 o'clock umbilical. The volume of the infiltrated solution varied between $500 \mathrm{ml}$ and $1000 \mathrm{ml}$. The infiltration was preferably carried out in the deep subcutaneous cellular tissue. 10 minutes after the infiltration of the lactated Ringer's solution and adrenaline, we proceed to liposuction. The liposuction must be carried out symmetrically, making fan strokes in the area where we intend to take the graft, taking care to distribute the strokes evenly.

The previously infiltrated solution allows the graft to be taken less traumatic, with less bleeding. This occurs for two reasons, the dissection of the vessels by the solution and the vasoconstrictor effect of adrenaline. The cannulas used were blunt, between 5 and $7 \mathrm{~mm}$ in diameter, with a number of holes between one and three, and a negative suction wall pressure of $350 \mathrm{mmHg}$ was used. The blunt tip of the cannulas helps prevent visceral injury. The abdominal liposuction is usually processed to obtain the definitive autologous adipose tissue graft. We used the technique described by Coleman [6], by centrifuging at 3000 rpm for 3 minutes. This technique is based on obtaining a dense graft, as Coleman initially described it for grafting in the facial area. After centrifugation, the lipoaspirate is separated into three components with a decreasing order of density. From highest to lowest density: serum from the tumescent infiltration solution with traces of blood and clots, connective tissue, adipose tissue, oil (non-viable adipose tissue). We discarded all of the components except the adipose tissue. The adipose tissue was transferred to new syringes to be grafted in the area of the breast to be corrected, trying to avoid manipulation or contact with air. The yield of liposuction, to obtain adipose tissue useful to be grafted, is usually $50 \%$ - $60 \%$. The adipose tissue concentrated and separated in syringes with a safety screw cap was infiltrated in the breast, in the area with the defect to be corrected. For this, incisions were made in the periphery of the breast of 1-2 $\mathrm{mm}$ (if they do not exceed this size, they can be non-sutured). A retreating infiltrator was carried out.

That is, the cannula was inserted, the tissue should be felt to be dissected, offering some resistance, and the plunger is pressed with the withdrawal of the cannula. The cannulas used were mostly $1 \mathrm{~mm}$ in diameter and some were $2 \mathrm{~mm}$, Coleman infiltration cannulas with a hole at the distal end and a blunt tip. The infiltration was performed in a clockwise direction, in several planes parallel to the chest wall, thus avoiding perpendicular infiltrations that could end in a pneumothorax. If we could see the final three-dimensional structure of the entire lipograft used, it would be like a honeycomb or lattice. We must over-correct the defect, achieving an appearance of the breast at the end of the intervention, with a volume greater than desired. A fundamental fact in the use of grafts is that they are nourished from the bed where they settle. For this reason, we must achieve fine cords of processed adipose tissue, surrounded by well vascularized healthy breast tissue. The thickness of the adipose graft "cords" deposited in the tissues by means of the cannulas, must guarantee a good contact surface between the receptor bed and the adipocytes, to enable their survival. This thickness is measured: It must be a maximum of $1 \mathrm{~mm}$. Up to 300 microns the survival of the graft is total, but even up to diameters of $1 \mathrm{~mm}$ the survival is very high.

By reviewing medical literature, we have collected the following variables:

a) Epidemiological data: Age at diagnosis / Smoker at diagnosis / Family history of BC (1st and 2nd degree relatives).

b) Reproductive data: Menarche / Pregnancies (number of deliveries) / Age of first delivery / Breastfeeding / Premenopause-Postmenopause at diagnosis / Use of hormonal contraceptive methods / Type of hormonal contraceptive method (oral, ring, patch, implant or IUD).

c) Tumor data: Date of CS / Histological type / Molecular subtypes / Grade / Vascular involvement / Presence of In Situ Carcinoma / Tumor size / Free surgical margins (the affected margin is defined when the infiltrating carcinoma reaches the ink (tumor on ink) and in the case of in situ component less than 2mm / Lymph node involvement / Number of affected lymph nodes / Number of resected lymph nodes.

d) LPF technique: Date of first LPF / Fat extraction location (abdominal, lower limb or mixed) / Volume of fat transferred / Time elapsed after CS (in months) / Subsequent asymmetries / Time of diagnosis of asymmetry after LPF (in months) / Patient satisfaction. We have assessed both posterior asymmetries and patient satisfaction through the subjective assessment of the follow-up transcribed in the medical history by the plastic surgeon. 
e) Complementary treatment data: Radiotherapy / Chemotherapy / AntiHer monoclonal antibody / Hormone therapy / Duration of Hormone therapy (less than 5 years or greater than or equal to 5 years).

f) Local tumor recurrence / Date of tumor recurrence / Time elapsed after CS (months) / Time elapsed after LPF (months) / Date of last follow-up (last imaging test performed in a follow-up period greater than or equal to 5 years) / Other data (relevant data on the case / control).

In the statistical analysis, IBM SPSS Statistics Version 24 has been used in the descriptive statistics part, for the rest of the analysis the R software version 3.5.5 https://www.R-project.org/ has been used. EPIDAT version 3.1 to support the conclusions of R. The statistical techniques used have been: Frequency tables, Contingency tables, Fisher's exact test, Pearson's Chi-square test, Shapiro-Wilks's normality tests, Var.test to compare variance, t.test to compare means, Nonparametric Mann- Whitney test. The graphs used have been: Boxplot or box plot or whisker graph and Barplot. The approval of the ethics committee is attached.

Table 1: Justification that cases and controls are comparable.

\begin{tabular}{|c|c|c|c|c|c|}
\hline \multirow[b]{2}{*}{ Ganglion affectation } & \multicolumn{2}{|c|}{ Cases $(n=20)$} & \multicolumn{2}{|c|}{ Controls $(n=57)$} & \multirow{2}{*}{$\frac{p \text {-value }}{1}$} \\
\hline & 11 & $55.00 \%$ & 30 & $52,63 \%$ & \\
\hline Menopause at diagnosis & 19 & $95.00 \%$ & 49 & $85.96 \%$ & 0.4322 \\
\hline \multicolumn{6}{|l|}{ Molecular Subtypes } \\
\hline Luminal A & 5 & $26.32 \%$ & 20 & $35.09 \%$ & 0.737 \\
\hline Luminal B Her positive & 4 & $21.05 \%$ & 8 & $14.04 \%$ & 0.7274 \\
\hline Luminal B Her negative & 9 & $47.37 \%$ & 25 & $43.86 \%$ & 1 \\
\hline Triple negative & 1 & $5.26 \%$ & 4 & $7.02 \%$ & 1 \\
\hline
\end{tabular}

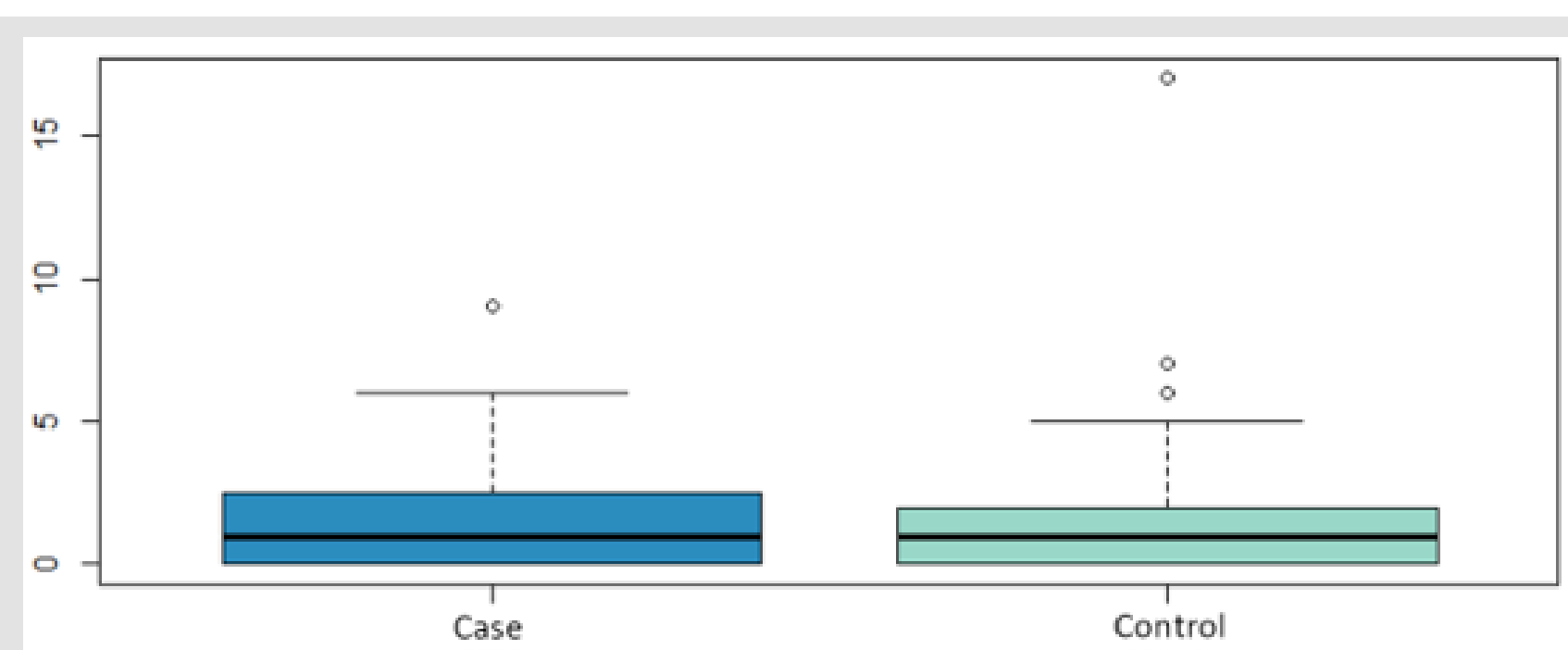

Figure 1: Number of lymph nodes affected for cases and controls. 


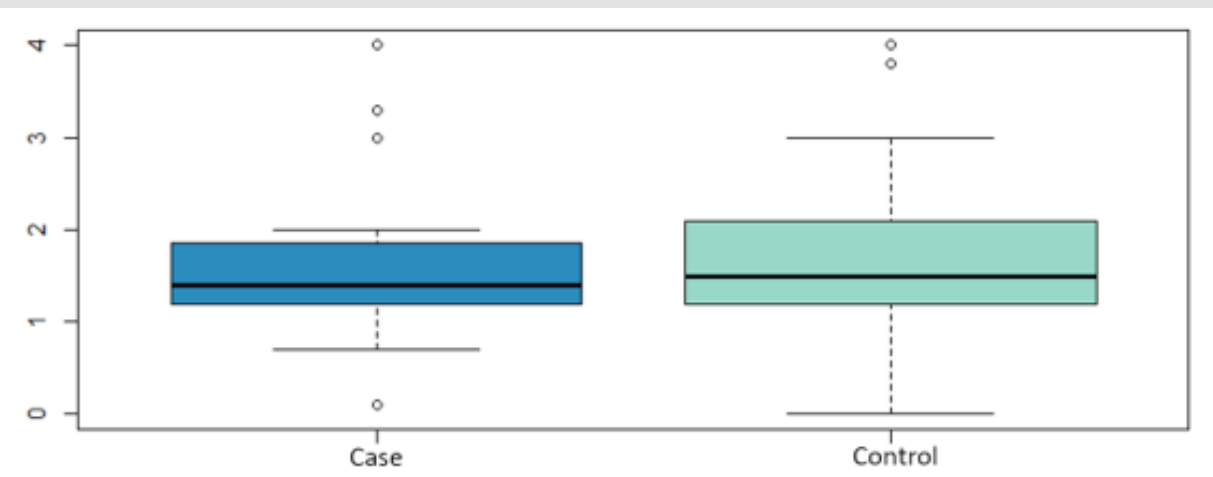

Figure 2: Boxplot of tumor size for cases and controls.

Fat donor areas were abdomen in $80 \%$ (16 patients), lower extremity in $10 \%$ (2 patients) or mixed in the remaining $10 \%$ (2 patients). The number of LPF sessions are: 1 LPF in $55 \%$ (11 patients), $2 \mathrm{LPF}$ in $25 \%$ (5 patients) and $3 \mathrm{LPF}$ in $20 \%$ (4 patients). In the 20 cases subjected to the LPF technique, the average volume of fat transferred is 290.5 cc. No local recurrences have been observed in the controls $(0 \%)$, however, there have been 4 local recurrences in the cases (20\%), a statistically significant difference with a $\mathrm{p}$ value of 0.0036 .

The influence on relapses of the variables: tumor size, vascular involvement, duration of hormonal therapy, use of chemotherapy has been analyzed, without finding statistically significant differences in any of them. Nor has the influence of the time elapsed since the date of CS and the date of the first LPF and the volume of fat transferred with the risk of local recurrence, as shown in (Figure 3 and Figure 4). Likewise, none of the four local recurrences had affected lymph nodes. $50 \%$ of the cases were satisfied with the result of the LPF technique, but in the remaining $50 \%$ there are no data to be able to assess the degree of satisfaction, due to which the factors that influence this item cannot be analyzed. Asymmetries have been observed in $20 \%$ of the patients undergoing LPF (4 patients), 8 have adequate symmetry ( $40 \%$ ) and in the remaining $8(40 \%)$ it has not been possible to assess the asymmetry due to lack of data in the medical history.

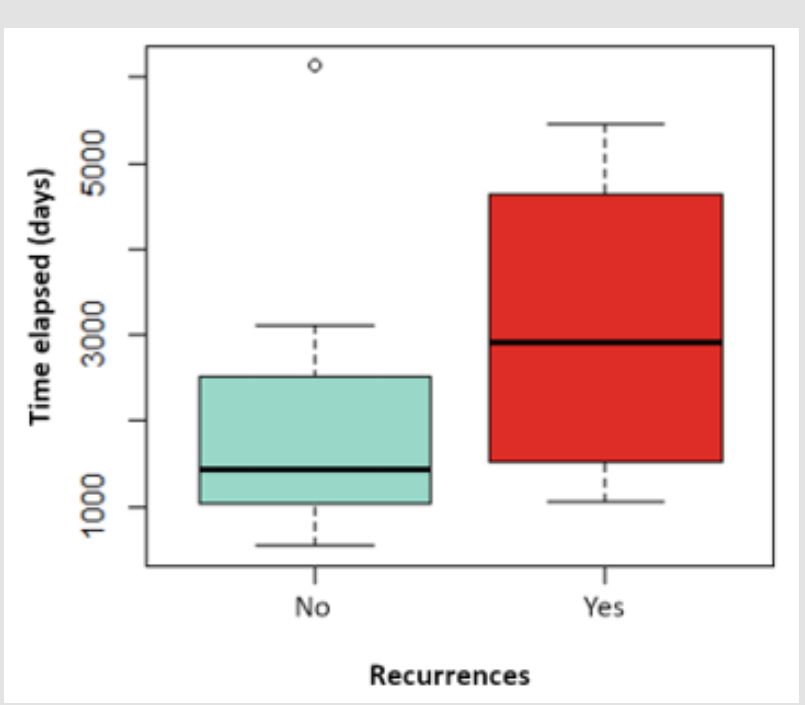

Figure 3: Time elapsed in days for women with recurrences or not. P value: 0.2485. 


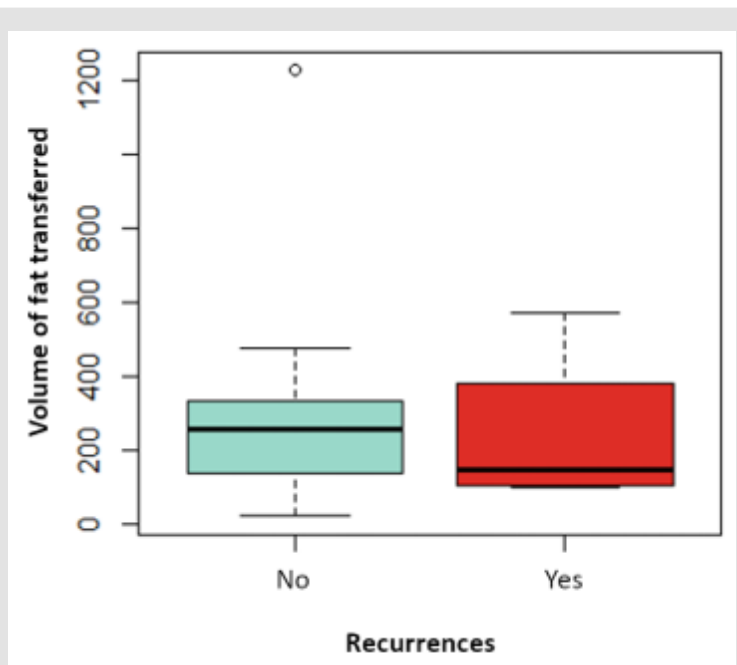

Figure 4: Boxplot of the variable volume of fat transferred depending on whether they have suffered recurrences in red or not in blue. P value: 0.689 .

\section{Discussion}

In our study, a $20 \%$ incidence of local recurrence have been evidenced in patients undergoing LPF after CS for BC: in contrast with Rigotti et al. [12] that found local recurrence of $6.5 \%$ (137 patients) after radical mastectomy with subsequent LPF. More recently, a meta-analysis, by Claro et al. [13] showed an incidence of local recurrence of $2.27 \%$, but in most recurrences the initial surgical procedure is mastectomy. In our study, LPF is a possible risk factor for local tumor recurrence. This link has not been described but most of the published articles present samples with patients undergoing both mastectomies and CS, and it is well known that in mastectomy the risk local recurrence rate is lower than in CS, $10 \%$ versus $39 \%$ [3], which may influence their final conclusions. There is also heterogeneity in the types of breast lesions, as not only invasive carcinomas are included, but carcinomas in situ (intraductal and lobular) are also introduced. Stumpf et al. [14] performed a study focusing only on CS and invasive BC and described 4 local recurrences (2.4\%) in the group of patients who underwent CS out of a total of 167 patients and no patient in the LPF group, 27 patients, had local recurrence during the study period. Although the differences are not statistically significant, they hypothesize that LPF does not influence local tumor recurrence. There are several differences respect to our study: the moment of insertion of the fat in the receiving area is carried out immediately in the case of Stumpf et al., while in our study it is done in a second time (one average of 5.77 years later); Likewise, the follow-up time is shorter in Stumpf et al. of 36 months, compared to a mean followup of our controls of 11.8 years.

In most studies, the patient follow-up time after performing LPF is 2 to 5 years, so the peak of late local recurrence that occurs from 5 to 8 years is not evaluated, while our work maintained the follow for at least 5 years, may be one of the causes for which our study locates these relapses compared to those previously published in the literature with shorter follow-up periods. An article has been published that does state that LPF is a risk factor for local recurrence. Petit et al. [15]. collected data from 321 patients that underwent surgery for a primary breast tumor and subsequently reconstructive LPF, they used 2 controls with similar characteristics that were not subjected to LPF were selected per study case and found 8 cases of local recurrence versus 19 recurrences in controls, not obtaining statistically significant results; However, in the group of patients with carcinoma in situ who underwent LPF, the local recurrence increased, this difference being statistically significant. Within this group, 3 cases occurred on mastectomy and 1 on CS. After this study, Petit et al. evaluated patients with carcinoma in situ exclusively in 2013, 6 local recurrences were observed in cases (18 $\%$ ) compared to 3 in controls (3\%), thus confirming an increased risk of local recurrence in these patients.

We have ruled out a series of confounding factors in the risk of development of local recurrence, such as lymphovascular involvement, lymph node involvement and tumor size, not showing statistically significant differences in recurrences. Other known risk factors are the degree and cancers with negative hormone receptors, our four relapses present luminal tumors A or B with Grade 1 or 2 of one of the relapses we have no data in this regard, so we cannot provide data with statistical significance. The percentage of satisfaction of our patients is high, reaching $50 \%$, but in the other $50 \%$ it has not been possible to assess it because data is not evidenced in the clinical records. Today it is known that necrosis of part of the graft usually leads to oily cysts, fibrosis, or replacement of the necrotized adipose tissue by new adipose tissue. This also has a filling effect, the extent to which this filling, which is not truly 
adipose tissue, behaves better or worse for the future is not clear. Finally, it is important to point out that there is a reabsorption of 40-50\% of the lipograft introduced into the breast, for two reasons: ischemia of the adipocytes in a bed that does not provide them with enough nutrients and due to the trauma suffered by the adipocytes with the intake of the lipograft when aspirating. Therefore, we must over-correct the defects, infiltrating $140 \%$ of the real volume that we want to obtain. But keeping in mind that a tissue with too much graft will be compressed, excessively expanded and worse perfused, causing ischemia of the adipocytes and a result opposite to that desired: loss of most of the lipograft. Likewise, the percentage of asymmetries is only $20 \%$, but in $40 \%$ of the cases there is no information in this regard.

The measurements of these variables are subjective since we perform them based on the evaluations made by the plastic surgeon in the postoperative follow-up. Only one study, Zocchi and Zuliani [16], uses an objective protocol to estimate patient satisfaction based on a 4-item scale (excellent, good, fair and insufficient). Although the lack of information in a large group of cases in our study does not allow us to draw conclusions based on statistical evidence, there have been multiple previous studies that have confirmed the aesthetic benefits and subsequent patient satisfaction, [17] demonstrate that with the use of LPF after CS of $\mathrm{BC}$ there is an improvement in irregularities, tissue consistency and increase in volume; A systematic review of 12 studies concludes that in 7 of them the volume gain after the use of LPF is confirmed with good or excellent results [18]. Our study has several limitations, such as the small sample size and its retrospective nature.

\section{Conclusion}

LPF may be a risk factor for local recurrence of breast cancer after conservative surgery, although more prospective studies with larger samples are needed to confirm the safety of LPF in this field.

\section{References}

1. F Bray, J Ferlay, I Soerjomataram, R L Siegel, L A Torre, et al. (2018) Global cancer statistics 2018: GLOBOCAN estimates of incidence and mortality worldwide for 36 cancers in 185 countries. CA Cancer J Clin 68(6): 394-424.

2. M Sant, Claudia Allemani, Mariano Santaquilani, Arnold Knijn, Francesca Marchesi, et al. (2009) EUROCARE-4. Survival of cancer patients diagnosed in 1995-1999. Results and commentary. Eur J Cancer 45(6): 931-991.
3. D A Mahvi, R Liu, M W Grinstaff, Y L Colson, C P Raut, et al. (2018) Local Cancer Recurrence: The Realities, Challenges, and Opportunities for New Therapies. CA Cancer J Clin 68(6): 488-505.

4. X Zhou, Y Li (2016) Local Recurrence after Breast-Conserving Surgery and Mastectomy Following Neoadjuvant Chemotherapy for Locally Advanced Breast Cancer-a Meta- Analysis. Breast Care 11(5): 345-351.

5. Y Belkacemi, N E Hanna, C Besnard, S Majdoul, J Gligorov, et al. (2018) Local and regional breast cancer recurrences: Salvage therapy options in the New Era of molecular subtypes. Frontiers in Oncology 8: 112.

6. J Masia, D Bordoni, G Pons, C Liuzza, F Castagnetti, G Falco, et al. (2015) Oncological safety of breast cancer patients undergoing free-flap reconstruction and lipofilling. Eur J Surg Oncol 41(5): 612-616.

7. L Tessitore, B Vizio, O Jenkins, I De Stefano, C Ritossa, et al. (2000) Leptin expression in colorectal and breast cancer patients. Int J Mol Med 5(4): 421-426.

8. M Ishikawa, J Kitayama, H Nagawa (2004) Enhanced expression of leptin and leptin receptor (OB-R) in human breast cancer. Clin Cancer Res 10(13): 4325-4331.

9. N Rahimi, R Saulnier, B Elliott, T Nakamura, M Park, et al. (1994) Role of Hepatocyte Growth Factor in Breast Cancer: A Novel Mitogenic Factor Secreted by Adipocytes. DNA Cell Biol 13(12): 1189-1197.

10. N C Direkze, Kairbaan Hodivala-Dilke, Rosemary Jeffery, Toby Hunt, Richard Poulsom, et al. (2004) Bone marrow contribution to tumorassociated myofibroblasts and fibroblasts. Cancer Res 64(23): 84928495.

11. J A Klein, D R Jeske (2016) Estimated Maximal Safe Dosages of Tumescent Lidocaine. Anesth Analg 122(5): 1350-1359.

12. G Rigotti, Alessandra Marchi, Paolo Stringhini, Guido Baroni, Mirco Galiè, et al. (2010) Determining the oncological risk of autologous lipoaspirate grafting for post-mastectomy breast reconstruction. Aesthetic Plast. Surg 34(4): 475-480.

13. F Claro, J C A Figueiredo, A G Zampar, A M Pinto-Neto (2012) Applicability and safety of autologous fat for reconstruction of the breast. British Journal of Surgery 99(6): 768-780.

14. C C Stumpf, Jorge Villanova Biazus, Fernando Schuh Ângela Erguy Zucatto, Rodrigo Cericatto, José Antônio Crespo Cavalheiro, et al. (2017) Reconstrução imediata com enxerto autólogo de gordura: Influência na recorrência local de câncer de mama. Rev Col Bras Cir 44(2): 179-186.

15. J Y Petit, E Botteri, V Lohsiriwat, M Rietjens , F De Lorenzi, et al. (2012) Locoregional recurrence risk after lipofilling in breast cancer patients. Ann Oncol 23(2): 582-588.

16. M L Zocchi, F Zuliani (2008) Bicompartmental breast lipostructuring. Aesthetic Plast Surg 32(2): 313-328.

17. I Schultz, A Lindegren, M Wickman (2012) Improved shape and consistency after lipofilling of the breast: Patients' evaluation of the outcome. Journal of Plastic Surgery and Hand Surgery 46(2): 85-90.

18. RD Largo, Laurent AHTchang, Valentin Mele, Arnaud Scherberich, Yves Harder, et al. (2014) Efficacy, safety and complications of autologous fat grafting to healthy breast tissue: A systematic review. Journal of Plastic, Reconstructive and Aesthetic Surgery 67(4): 437-448. 
ISSN: 2574-1241

DOI: 10.26717/BJSTR.2021.34.005554

Peña-Fernández M. Biomed J Sci \& Tech Res

(C) (P) This work is licensed under Creative BY Commons Attribution 4.0 License

Submission Link: https://biomedres.us/submit-manuscript.php

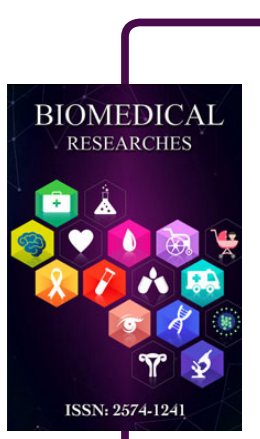

Assets of Publishing with us

- Global archiving of articles

- Immediate, unrestricted online access

- Rigorous Peer Review Process

- Authors Retain Copyrights

- Unique DOI for all articles

https://biomedres.us/ 